\title{
Effect of Statin Treatment in Patients With Advanced Carotid Atherosclerosis: An Observational Outcome Study
}

\author{
Ansgar Adams $^{\mathrm{a}, \mathrm{d}}$, Waldemar Bojara ${ }^{\mathrm{b}}$, Michel Romanens ${ }^{\mathrm{c}}$
}

\begin{abstract}
Background: Advanced atherosclerosis of the carotid artery is associated with a high risk of cardiovascular disease. The aim of the study was to investigate whether treatment with statins improved the prognosis.
\end{abstract}

Methods: Sum of all plaque areas (total plaque area (TPA)) and the maximum plaque thickness were determined in healthy subjects using ultrasound. We compared the outcome in subjects with advanced atherosclerosis of the carotid artery (type III-IV $b$ finding) with and without statin treatment. The follow-up was recorded during followup examinations as part of preventive occupational health examinations or by personal communication.

Results: In 7,106 subjects aged 35 - 65 years $(50 \pm 8$ years, $43 \%$ women), we found 669 subjects with advanced atherosclerosis of the carotid artery (type III-IV b finding). A follow-up was available for $640(95.4 \%)$ subjects. In these subjects $(54 \pm 8$ years, $20.4 \%$ women), 94 ( $88 \mathrm{men}$ ) had cardiovascular events (35 myocardial infarctions, 13 bypass operations, 32 stent implantations, and 14 strokes) with a mean follow-up time of $3.9(1-12)$ years. Two hundred sixty subjects were treated with a statin, while 339 received no statin. Fourteen cardiovascular events occurred in the treated group (eight stent implantations, two heart attacks, two bypass operations, and two strokes). In the untreated group, 80 cardiovascular events occurred (12 strokes, 11 bypass operations, 33 heart attacks, and 24 stent implantations). The event rate was $5.4 \%$ for the subjects treated with a statin and $23.6 \%$ for the untreated subjects. Both groups were well matched for the baseline presence of cardiovascular risk factors.

Conclusion: Statin treatment in subjects with advanced atheroscle-

Manuscript submitted August 22, 2021, accepted October 4, 2021

Published online November 5, 2021

aBAD Gesundheitsvorsorge und Sicherheitstechnik GmbH Zentrum Koblenz, Koblenz, Germany

${ }^{b}$ Medizinische Klinik Kardiologie Koblenz, Gemeinschaftsklinikum Kemperhof II, Koblenz, Germany

${ }^{c}$ Vascular Risk Foundation (Varifo), Olten, Switzerland

${ }^{\mathrm{d} C o r r e s p o n d i n g ~ A u t h o r: ~ A n s g a r ~ A d a m s, ~ B A D ~ H e a l t h ~ C a r e ~ a n d ~ S a f e t y ~ T e c h-~}$ nology Centre GmbH, Koblenz, Germany.

Email: ansgar.adams@bad-gmbh.de

doi: https://doi.org/10.14740/cr1318 rosis of the carotid artery (type III-IV b finding on ultrasound) significantly improves the prognosis in a non-randomized observational cohort study.

Keywords: Total plaque area; Carotid ultrasound; Cardiovascular risk; Coronary disease

\section{Introduction}

It is known from previous studies that advanced atherosclerosis of the carotid artery is associated with an increased risk of cardiovascular disease [1-8]. By measuring all plaque areas (total plaque area (TPA)) and the maximum plaque thickness on the carotid artery, people with a high cardiovascular risk can be identified [9-11]. The examination method was validated by measuring the TPA and the maximum plaque thickness in 500 patients 1 day before a planned coronary angiography regardless of the indication. Four types could be defined in the ultrasound.

With a type III and IV b carotid plaque on ultrasound, we found the presence of coronary heart disease defined by a coronary angiogram in $82.2 \%$ [9].

\section{Materials and Methods}

In 4,076 healthy men and 3,030 healthy women aged $35-65$ years, the sum of all plaque areas (TPA) and the maximum plaque thickness were determined in an occupational medical setting using ultrasound. A transportable ultrasound device from Kontron Medical, type Imagic Agile, with a $10 \mathrm{MHz}$ linear transducer was used. The measurement method was carried out as previously published $[9,10]$. A low risk corresponds to a type I and type II a finding on ultrasound, an intermediate risk to a type II $b$ and IV a finding and a high risk to a type III and IV $b$ finding [11]. All subjects with a type III or IV $b$ finding received a recommendation for their family doctor to initiate a therapy with a statin.

We compared the outcome in subjects with advanced atherosclerosis of the carotid artery (type III-IV $b$ finding) with and without statin treatment. The follow-up was recorded during follow-up examinations as part of preventive occupational health examinations. The evaluation of all investigations was 
Table 1. Baseline Characteristics of All Subjects With Type III and IV b Finding in Ultrasound

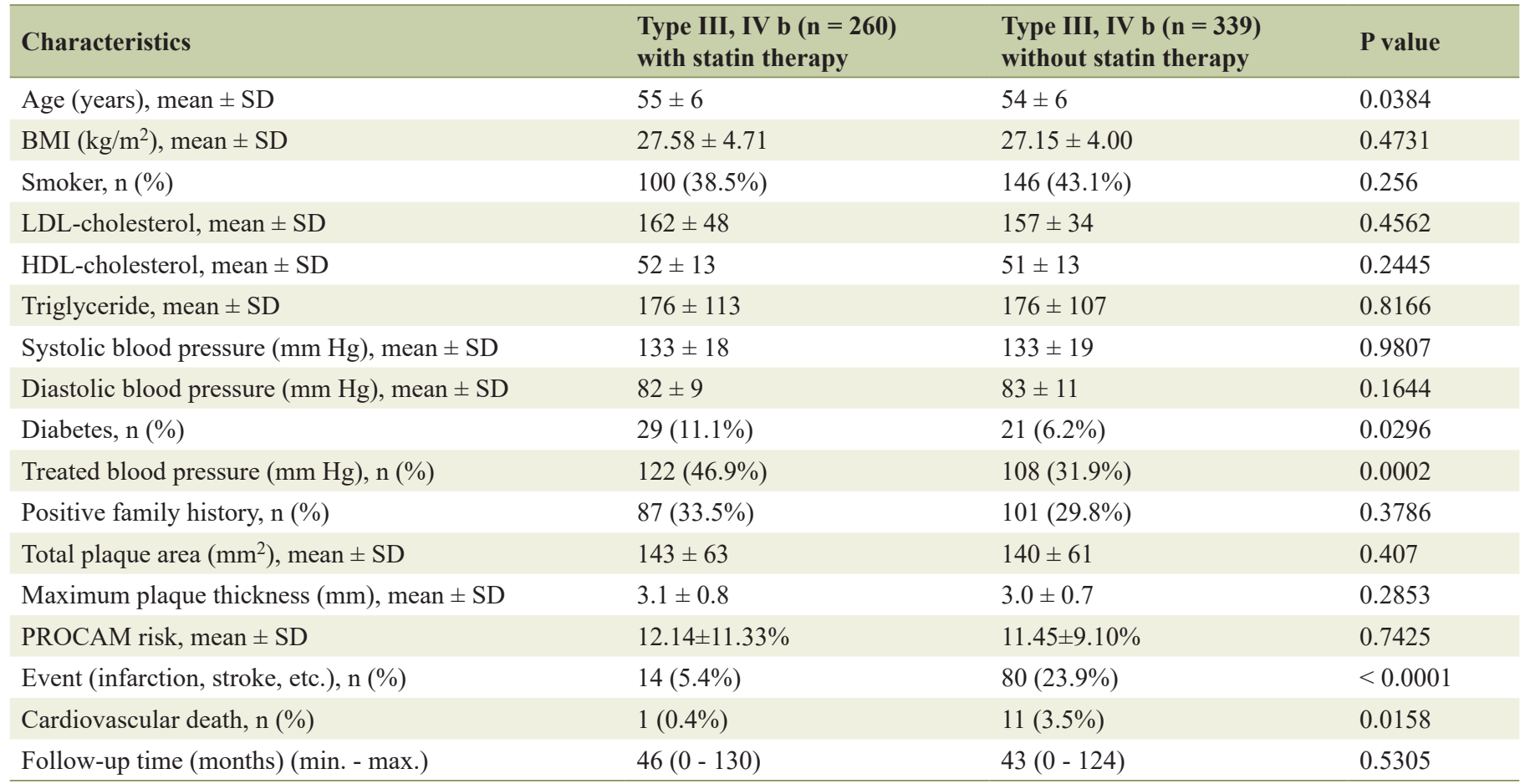

BMI: body mass index; LDL: low-density lipoprotein; HDL: high-density lipoprotein; PROCAM: Prospective Cardiovascular Munster Study for Fatal and Non-Fatal Myocardial Infarction; SD: standard deviation.

carried out with the approval of the responsible ethics committee. This study was conducted in compliance with the ethical standards of the responsible institution on human subjects as well as with the Helsinki Declaration.

As part of their occupational health check-ups, we asked patients to inform us about the occurrence of cardiovascular events (myocardial infarction, percutaneous transluminal coronary angioplasty (PTCA), bypass surgery (CABG) or stroke). Whenever possible we received clinical records from treating physicians. It was investigated whether statin treatment in subjects with advanced atherosclerosis of the carotid artery (type III-IV $b$ finding on ultrasound) leads to an improvement in the prognosis. Statin users reported statin use on a daily level during the whole follow-up time. Statin non-users were not prescribed statins during the whole observation time. There were no sporadic statin users. Effect of statins on low-density lipoprotein (LDL) cholesterol was not available. Further, we did not record changes in other cardiovascular risk factors such as smoking, hypertension, or new onset of diabetes mellitus. Further, we obtained a follow-up in some patients without highrisk carotid plaque.

\section{Results}

Type III and IV b findings were found in 669 subjects (135 women). A follow-up was available for 640 (95.5\%) subjects. In these subjects ( $54 \pm 8$ years, $20.4 \%$ women), 94 (88 men) had cardiovascular events (35 myocardial infarctions, 13 by- pass operations, 32 stent implantations, and 14 strokes), with a mean follow-up time of $3.9(1-12)$ years. Two hundred sixty subjects were treated with a statin, while 339 received no statin. No data on statin treatment were available for 41 subjects. These were excluded. The baseline data were almost the same in both groups, especially the Prospective Cardiovascular Munster Study for Fatal and Non-Fatal Myocardial Infarction (PROCAM) risk $(\mathrm{P}=0.7425)$.

Table 1 shows the clinical baseline characteristics of both groups.

Fourteen cardiovascular events occurred in the treated group (eight stent implantations, two heart attacks, two bypass operations, and two strokes). In the untreated group, 80 cardiovascular events occurred (12 strokes, 11 bypass operations, 33 heart attacks, and 24 stent implantations). The event rate was $5.4 \%$ for the subjects treated with a statin and $23.6 \%$ for the untreated subjects. Nine patients died of a heart attack, and three of stroke (11 were not treated with a statin).

In the low-risk group (type I, II a, $\mathrm{n}=5,712$ ), follow-up was complete in $34.6 \%$ of subjects with a mean follow-up time of 4.9 years ( 1 - 12 years), and there were 11 cardiovascular events (eight myocardial infarctions, two bypass operations, and one stent implantation). In the intermediate-risk group (type IIb, IV a, $\mathrm{n}=725$ ), follow-up was complete in $47.3 \%$ of subjects with a mean follow-up time of 4.3 years ( 1 - 12 years), and 10 cardiovascular events occurred (three myocardial infarctions, three bypass operations, two stent implantations, and two strokes), with one death [10]. Figure 1 shows unadjusted event prediction results for type III-IV $b$ finding in ultrasound 


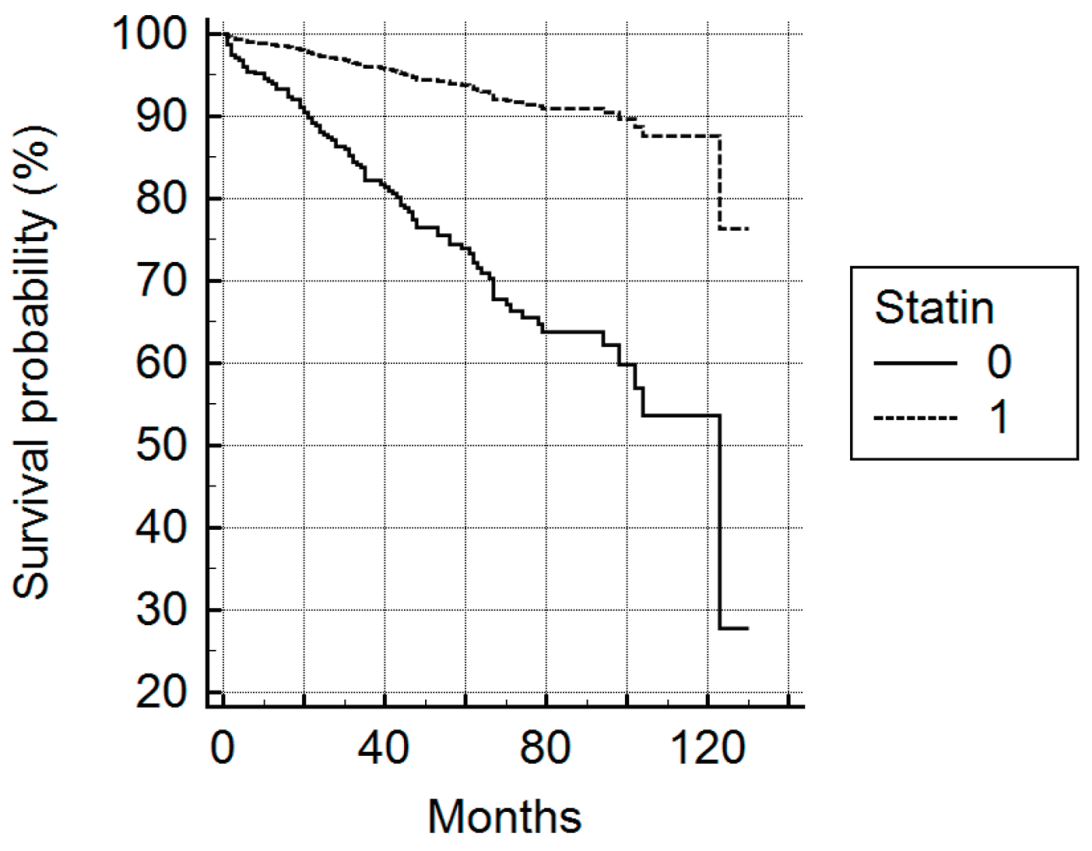

Figure 1. Unadjusted HRs for primary outcome (all events) associated with advanced atherosclerosis on carotid artery (type III-IV $b$ finding) as a function of statin use. HR: hazard ratio. $P<0.0001$.

with and without statin therapy.

\section{Discussion}

Atherosclerosis is an inflammatory disease of the arteries and continues to be the most common cause of death in industrialized nations.

Fatty streaks can be found in arteries already at the age of 20 years, and progression of atherosclerosis often remains undetected until clinical events such as acute coronary syndrome (ACS), chronic coronary syndrome (CCS), apoplexy, and peripheral artery disease (PAD) occur.

Because traditional risk equations such as PROCAM or SCORE show frequently low risk in patients with advanced carotid atherosclerosis [12], it is important to identify and treat advanced carotid atherosclerosis, a surrogate for cardiovascular risk, in the subclinical stage. We found advanced carotid atherosclerosis in $9 \%$ of subjects at baseline and in these, $55.2 \%$ had a PROCAM risk below 10\%. Therefore, PROCAM, at least in patients aged $35-65$ years, does not appear to reliably identify high-risk patients defined by extensive carotid plaque formation.

Both the ESC Guidelines on Cardiovascular Disease Prevention in Clinical Practice of 2016 and the ESC Guidelines for the Management of Dyslipidemia 2019 [13, 14] classify people with carotid plaques as having a very high risk, but without quantifying the plaque burden.

There are several plaque studies that show that advanced atherosclerosis of the carotid artery with high TPA is associated with a high risk of events (Tromso study, BioImage study, CAVES-CAVA study, Spence 2002, MESA study, NOMAS study, Adams 2020, and ARCO study 2021 [1-11]) and therapy with statins improves the prognosis [8, 15-19].

As early as 2010, Spence formulated "Treating arteries instead of risk factors. A paradigm change in management of atherosclerosis" [15]. Our results are consistent with these studies and support the paradigm shift formulated by Spence.

Although this is not a randomized study, the results suggest that patients with advanced atherosclerosis (type III, IV b finding) should be treated with a statin, eventually regardless of the level of LDL cholesterol in line with the postulate "treating arteries instead of risk factors".

\section{Conclusions}

Statin treatment in subjects with advanced atherosclerosis of the carotid artery (type III-IV $b$ finding on ultrasound) significantly improved prognosis as assessed by our observational cohort study. Since most patients with advanced carotid atherosclerosis have a low PROCAM risk, statin intervention is unlikely to be tailored correctly to patients who would derive the largest prognostic benefits from preventive therapy.

\section{Acknowledgments}

None to declare.

\section{Financial Disclosure}

This study has not been supported by any external funding. 


\section{Conflict of Interest}

The authors declare that there is no conflict of interest.

\section{Informed Consent}

All patients provided informed consent.

\section{Author Contributions}

WB and MR discussed the approach and the findings of this study with AA intensively, and gave AA valuable feedback. All examinations have been done by AA.

\section{Data Availability}

Any inquiries regarding supporting data availability of this study should be directed to the corresponding author.

\section{Abbreviations}

TPA: total plaque area (carotid plaque); PROCAM: Prospective Cardiovascular Munster Study for Fatal and Non-Fatal Myocardial Infarction

\section{References}

1. Belcaro G, Nicolaides AN, Ramaswami G, Cesarone MR, De Sanctis M, Incandela L, Ferrari P, et al. Carotid and femoral ultrasound morphology screening and cardiovascular events in low risk subjects: a 10-year followup study (the CAFES-CAVE study(1)). Atherosclerosis. 2001;156(2):379-387.

2. Johnsen SH, Mathiesen EB, Joakimsen O, Stensland E, Wilsgaard T, Lochen ML, Njolstad I, et al. Carotid atherosclerosis is a stronger predictor of myocardial infarction in women than in men: a 6-year follow-up study of 6226 persons: the Tromso Study. Stroke. 2007;38(11):28732880.

3. Mathiesen EB, Johnsen SH, Wilsgaard T, Bonaa KH, Lochen ML, Njolstad I. Carotid plaque area and intima-media thickness in prediction of first-ever ischemic stroke: a 10-year follow-up of 6584 men and women: the Tromso Study. Stroke. 2011;42(4):972-978.

4. Inaba Y, Chen JA, Bergmann SR. Carotid plaque, compared with carotid intima-media thickness, more accurately predicts coronary artery disease events: a metaanalysis. Atherosclerosis. 2012;220(1):128-133.

5. Rundek T, Arif H, Boden-Albala B, Elkind MS, Paik MC, Sacco RL. Carotid plaque, a subclinical precursor of vascular events: the Northern Manhattan Study. Neurology. 2008;70(14):1200-1207.

6. Baber U, Mehran R, Sartori S, Schoos MM, Sillesen H,
Muntendam P, Garcia MJ, et al. Prevalence, impact, and predictive value of detecting subclinical coronary and carotid atherosclerosis in asymptomatic adults: the BioImage study. J Am Coll Cardiol. 2015;65(11):1065-1074.

7. Romanens M, Adams A, Sudano I, Bojara W, Balint S, Warmuth W, Szucs TD. Prediction of cardiovascular events with traditional risk equations and total plaque area of carotid atherosclerosis: The Arteris Cardiovascular Outcome (ARCO) cohort study. Prev Med. 2021;147:106525.

8. Spence JD, Eliasziw M, DiCicco M, Hackam DG, Galil R, Lohmann T. Carotid plaque area: a tool for targeting and evaluating vascular preventive therapy. Stroke. 2002;33(12):2916-2922.

9. Adams A, Bojara W. [Prediction of coronary artery stenosis by measurement of total plaque area and thickness versus intima media thickness of the carotid artery]. Herz. 2015;40(5):817-822.

10. Adams A, Bojara W, Schunk K. Early diagnosis and treatment of coronary heart disease in asymptomatic subjects with advanced vascular atherosclerosis of the carotid artery (Type III and IV b Findings Using Ultrasound) and risk factors. Cardiol Res. 2018;9(1):22-27.

11. Adams A, Bojara W, Romanens M. The determination of the plaque burden on the carotid artery with ultrasound significantly improves the risk prediction in middle-aged subjects compared to PROCAM: an outcome study. Cardiol Res. 2020;11(4):233-238.

12. Romanens M, Mortensen MB, Sudano I, Szucs T, Adams A. Extensive carotid atherosclerosis and the diagnostic accuracy of coronary risk calculators. Prev Med Rep. 2017;6:182-186.

13. Piepoli MF, Hoes AW, Agewall S, Albus C, Brotons C, Catapano AL, Cooney MT, et al. 2016 European Guidelines on cardiovascular disease prevention in clinical practice: The Sixth Joint Task Force of the European Society of Cardiology and Other Societies on Cardiovascular Disease Prevention in Clinical Practice (constituted by representatives of 10 societies and by invited experts) Developed with the special contribution of the European Association for Cardiovascular Prevention \& Rehabilitation (EACPR). Eur Heart J. 2016;37(29):2315-2381.

14. Mach F, Baigent C, Catapano AL, Koskinas KC, Casula M, Badimon L, Chapman MJ, et al. 2019 ESC/EAS Guidelines for the management of dyslipidaemias: lipid modification to reduce cardiovascular risk. Eur Heart J. 2020;41(1):111-188.

15. Spence JD, Hackam DG. Treating arteries instead of risk factors: a paradigm change in management of atherosclerosis. Stroke. 2010;41(6):1193-1199.

16. Korcarz CE, DeCara JM, Hirsch AT, Mohler ER, Pogue B, Postley J, Tzou WS, et al. Ultrasound detection of increased carotid intima-media thickness and carotid plaque in an office practice setting: does it affect physician behavior or patient motivation? J Am Soc Echocardiogr. 2008;21(10):1156-1162.

17. Naslund U, Ng N, Lundgren A, Fharm E, Gronlund C, Johansson H, Lindahl B, et al. Visualization of asymptomatic atherosclerotic disease for optimum cardiovascular prevention (VIPVIZA): a pragmatic, open-label, ran- 
domised controlled trial. Lancet. 2019;393(10167):133142.

18. Spence JD, Coates V, Li H, Tamayo A, Munoz C, Hackam DG, DiCicco M, et al. Effects of intensive medical ther- apy on microemboli and cardiovascular risk in asymptomatic carotid stenosis. Arch Neurol. 2010;67(2):180-186.

19. Spence JD. Stroke prevention: a lifetime of lessons. Stroke. 2020;51(7):2255-2262. 